\title{
BMJ Open Recent intimate partner violence against women and health: a systematic review and meta-analysis of cohort studies
}

\author{
Loraine J Bacchus, Meghna Ranganathan, Charlotte Watts, Karen Devries
}

To cite: Bacchus LJ, Ranganathan M, Watts C, et al. Recent intimate partner violence against women and health: a systematic review and meta-analysis of cohort studies. BMJ Open 2018;8:e019995. doi:10.1136/ bmjopen-2017-019995

- Prepublication history and additional material for this paper are available online. To view these files, please visit the journal online (http://dx.doi. org/10.1136/bmjopen-2017019995).

Received 9 0ctober 2017 Revised 21 May 2018 Accepted 24 May 2018
Check for updates

(C) Author(s) (or their employer(s)) 2018. Re-use permitted under CC BY-NC. No commercial re-use. See rights and permissions. Published by BMJ.

Department of Global Health and Development, Faculty of Public Health and Policy, London School of Hygiene \& Tropical Medicine, London, UK

Correspondence to

Dr Loraine J Bacchus;

Loraine.Bacchus@Ishtm.ac.uk

\begin{abstract}
Objective We reviewed cohort studies to determine the magnitude and temporal direction of the association between recent intimate partner violence (IPV) and a range of adverse health outcomes or health risk behaviours. Design Systematic review and meta-analysis. Methods Medline, EMBASE and PsycINF0 were searched from the first record to November 2016. Recent IPV was defined as occurring up to and including the last 12 months; all health outcomes were eligible for inclusion. Results were combined using random-effects metaanalysis.
\end{abstract}

Results 35 separate cohort studies were retrieved. Eight studies showed evidence of a positive association between recent IPV and subsequent depressive symptoms, with a pooled $\mathrm{OR}$ from five estimates of $1.76(95 \% \mathrm{Cl} 1.26$ to $2.44, I^{2}=37.5 \%, p=0.172$ ). Five studies demonstrated a positive, statistically significant relationship between depressive symptoms and subsequent IPV; the pooled OR from two studies was $1.72\left(95 \% \mathrm{Cl} 1.28\right.$ to $2.31, \mathrm{I}^{2}=0.0 \%$, $\mathrm{p}=0.752$ ). Recent IPV was also associated with increased symptoms of subsequent postpartum depression in five studies $(\mathrm{OR}=2.19,95 \% \mathrm{Cl} 1.39$ to $3.45, \mathrm{p}=0.000)$, although there was substantial heterogeneity. There was some evidence of a bidirectional relationship between recent IPV and hard drug use and marijuana use, although studies were limited. There was no evidence of an association between recent IPV and alcohol use or sexually transmitted infections (STIS), although there were few studies and inconsistent measurement of alcohol and STIs.

Conclusions Exposure to violence has significant impacts. Longitudinal studies are needed to understand the temporal relationship between recent IPV and different health issues, while considering the differential effects of recent versus past exposure to IPV. Improved measurement will enable an understanding of the immediate and longer term health needs of women exposed to IPV. Healthcare providers and IPV organisations should be aware of the bidirectional relationship between recent IPV and depressive symptoms.

PROSPERO registration number CRD42016033372.

\section{BACKGROUND}

Worldwide, almost a third (30\%) of all women who have been in a relationship have experienced physical and/or sexual violence by their intimate partner. ${ }^{12}$ Women's lifetime exposure to intimate partner violence (IPV)

\section{Strengths and limitations of this study}

This is the first systematic review of cohort studies to measure the magnitude of the association and temporal direction between recent exposure to intimate partner violence (IPV) and health outcomes.

- As the review considers a broad range of outcomes, we identified gaps in the evidence base, including a need for cohort studies on recent IPV and non-communicable diseases such as cardiovascular disease hypertension and obesity, as well as post-traumatic stress disorder and anxiety disorder.

- Due to the large number of abstracts retrieved and the limited timeframe for the review, we were not able to employ double screening of abstracts; however, two researchers conducted the review of the final set of full-text papers, with a third reviewer of all full text papers where there was uncertainty about their inclusion.

- As some studies measured the outcome variable (either IPV or the health condition) continuously, it was not possible to combine all measures of effect, which limited the number of studies in the meta-analysis.

- It was not possible to quantitatively assess publication bias, as too few studies were in the meta-analysis of each health condition.

is associated with myriad health outcomes. Systematic reviews of longitudinal data find that women who have been physically and/ or sexually abused by their partner at some point in their life are twice as likely to have an abortion, twice as likely to suffer from depression, and in some regions are 1.5 times more likely to acquire HIV compared with women who have not experienced IPV. ${ }^{2}$ Not surprisingly, given its high prevalence and adverse health effects, lifetime exposure to IPV is estimated to result in a high burden of disease. IPV is the second most common risk factor for disability-adjusted life years globally in women aged 20-24 years. ${ }^{3}$

In our previous systematic reviews, we began to explore the relationships between 'ever' exposure to IPV and depressive symptoms and alcohol use, which revealed evidence of 
a bidirectional association. Devries $e t a l^{4}$ found evidence suggestive of an association between IPV and incident depressive symptoms ( $\mathrm{OR}=1.97,95 \%$ CI 1.56 to 2.48$)$, as well as an association in the reverse direction between depressive symptoms and incident IPV (OR=1.93, 95\% CI 1.51 to 2.48). In another systematic review the authors found increased odds of alcohol use following IPV ( $\mathrm{OR}=1.25,95 \% \mathrm{CI} 1.02$ to 1.52$)$ and increased odds of IPV following alcohol use (OR=1.27, 95\% CI 1.07 to 1.52$).^{5}$

Although available evidence finds important associations between IPV and a range of mental and physical health outcomes, the nature of the associations is not always clear. It is possible that exposure to IPV results in subsequent mental and physical health outcomes, that different mental and physical health conditions increase the risk of subsequent IPV, or that a bidirectional relationship is present.

Both IPV and some associated health outcomes, such as depression, anxiety and substance abuse, are chronic, episodic conditions, which can occur with varying frequency over longer time periods. Studies that measure lifetime exposure to IPV therefore hide the complexity of the relationship between IPV and mental and physical health outcomes. This is because estimates of 'ever' exposure to IPV are heterogeneous, and may include anything from past year, before the past year and more distant experiences of IPV. Recent violence may lead to more severe health outcomes, but this may be influenced by duration and severity, for example, recent violence with no history versus recent violence experienced as part of ongoing historical abuse.

In the current systematic review, we build on this by closely examining the issue of temporality with regard to recent exposure to IPV and a broader range of health outcomes. In this paper we aim to (1) review what health outcomes have been examined in cohort studies of recent IPV ('recent' defined here as IPV experienced up to and including the last 12 months); (2) quantify the magnitude of the association between IPV and different health outcomes; and (3) examine the temporal direction of IPV and health outcomes.

\section{METHODS}

A systematic review protocol was registered on PROSPERO on 18 March 2016 (CRD42016033372) and is available at http://www.crd.york.ac.uk/PROSPERO/display_ record.php?ID=CRD42016033372.

\section{Literature searches}

We searched Medline, EMBASE and PsycINFO from the first record up to 27 January 2016 (with an updated search conducted in November 2016). Terms for IPV were adapted from a previous systematic review on the prevalence of IPV and health outcomes, which was conducted for the 2010 Global Burden of Disease of IPV. ${ }^{1}$ Controlled vocabulary terms and text words related to longitudinal studies were used for each database. In order to ensure a wide yield of studies, terms for specific health outcomes were not included. An example of search strategy appears in online supplementary appendix 1 . Reference list screening was undertaken for key systematic review papers. One study was included from a systematic review on IPV and perinatal mental health disorders published in 2017, which we identified while this paper was under review.

\section{Inclusion criteria}

- English-language publications.

- Longitudinal studies reporting on female participants aged 15 and over were considered. Studies were deemed longitudinal if either the exposure or the outcome was measured on at least two occasions.

- Studies where IPV was conceptualised as the independent variable, or where IPV was the dependent variable, in order to capture any evidence of bidirectional causality.

- All author definitions of recent IPV victimisation that occurred up to and including 12 months prior.

- All author definitions of women-related health outcomes that were measured on at least two occasions.

A 12-month cut-off period was chosen for recent IPV as this is the most commonly used period for prevalence estimates, it is consistent with internationally recognised IPV measures, ${ }^{67}$ and has been used in a number of intervention studies for IPV. ${ }^{8-10}$

\section{Screening and data extraction}

Records were initially screened by one reviewer (LJB), and studies not meeting the inclusion criteria were removed. Full-text articles were reviewed by one reviewer (LJB), and where there was uncertainty about the inclusion of an article it was referred to the senior author $(\mathrm{KD})$. The final set of full-text articles was formally appraised by two reviewers (LJB and MR). Data were extracted and entered into an Excel spreadsheet by one reviewer (LJB). The study selection process including the number of studies, abstracts and full texts screened with reasons for exclusion is summarised in the flow chart in figure 1 .

\section{Quality appraisal}

The quality of each effect estimate was appraised and presented in table 1, which corresponds to the major relevant domains of potential bias in quality assessment tools. Consideration was given to whether definitions of IPV and health outcomes were based on valid, reliable measures. We considered whether studies controlled for potential confounders for two reasons. IPV and the health outcomes of interest commonly occur episodically over a period of time, and episodes of either that are incident over the study period may be a continuation of previous IPV or health outcomes. Therefore, we examined whether studies adjusted for time 1 levels (ie, at the beginning of the study period) of the outcome variable. Additionally, IPV and the health outcomes of interest are associated with demographic characteristics and other risk 


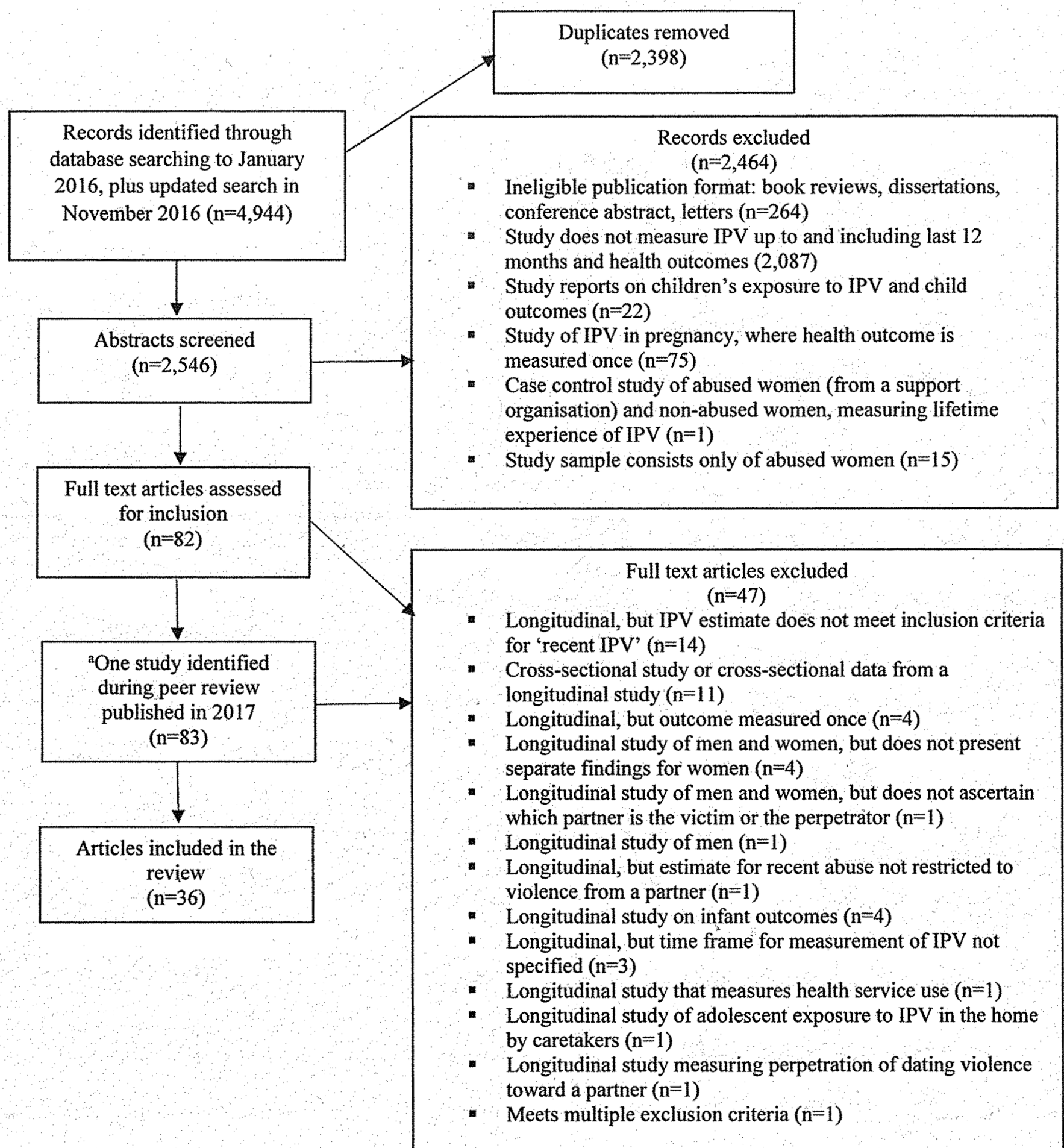

Figure 1 Flow of studies through review. ${ }^{a}$ New systematic review published in 2017 on domestic violence in pregnancy and perinatal mental health disorders identified by editorial team during the peer review of our paper. IPV, intimate partner violence.

factors that may explain the association between them, such as childhood sexual abuse. Due to the complexity of the potential causal pathways between IPV and the health outcomes, we did not specify a minimum set of confounders that should be adjusted for. Additionally, it has been noted that it is not always appropriate to adjust for baseline levels of an outcome variable in longitudinal studies. When exposures are associated with baseline health status, bias can arise if change in health status preceded baseline assessment or if the dependent variable measurement is unreliable or unstable. ${ }^{11}$ However, we recorded whether key variables were adjusted for and examined the results in the light of these adjustments. Information was also extracted in relation to mode of administration of surveys, length of follow-up, number of waves and attrition rates. 


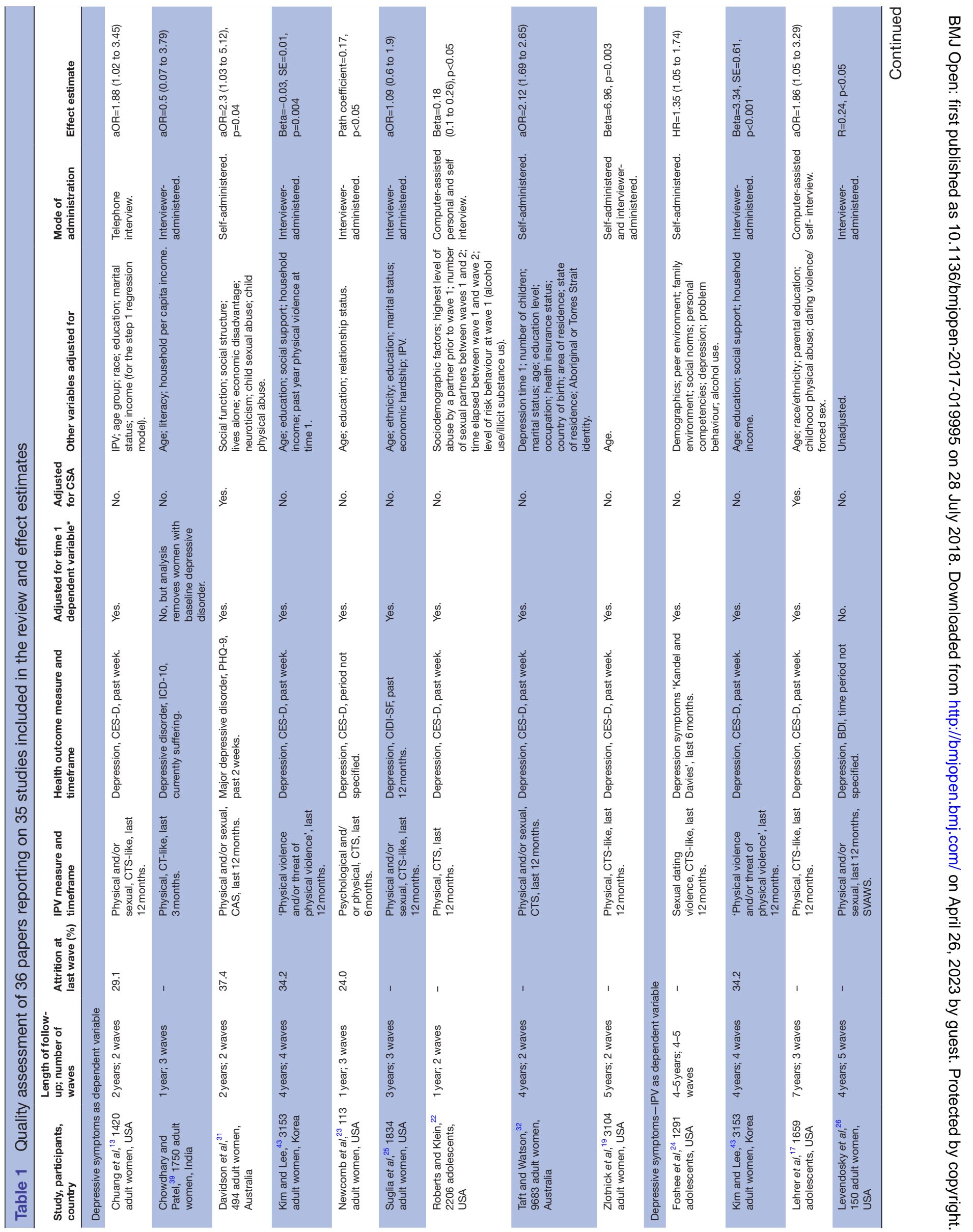




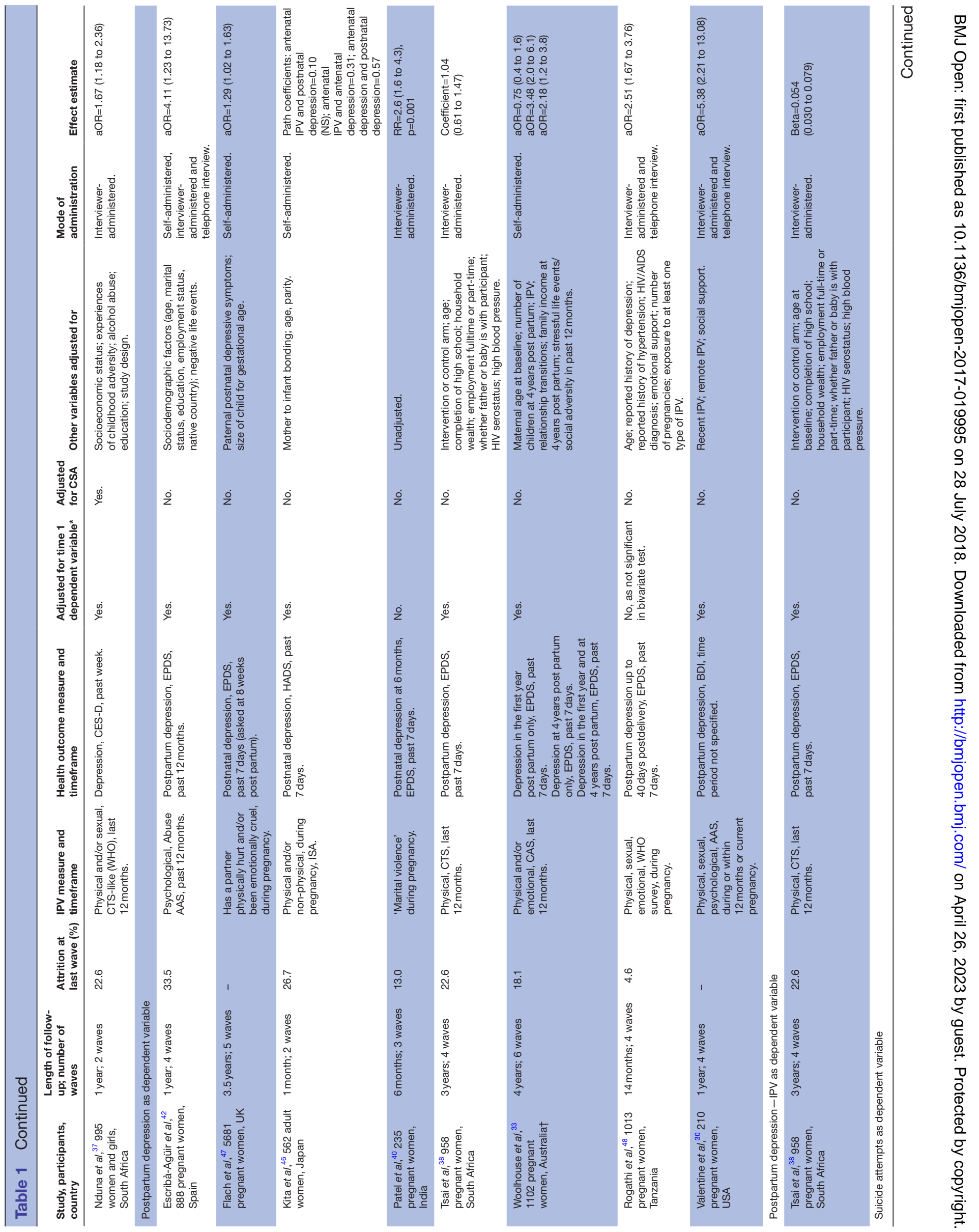




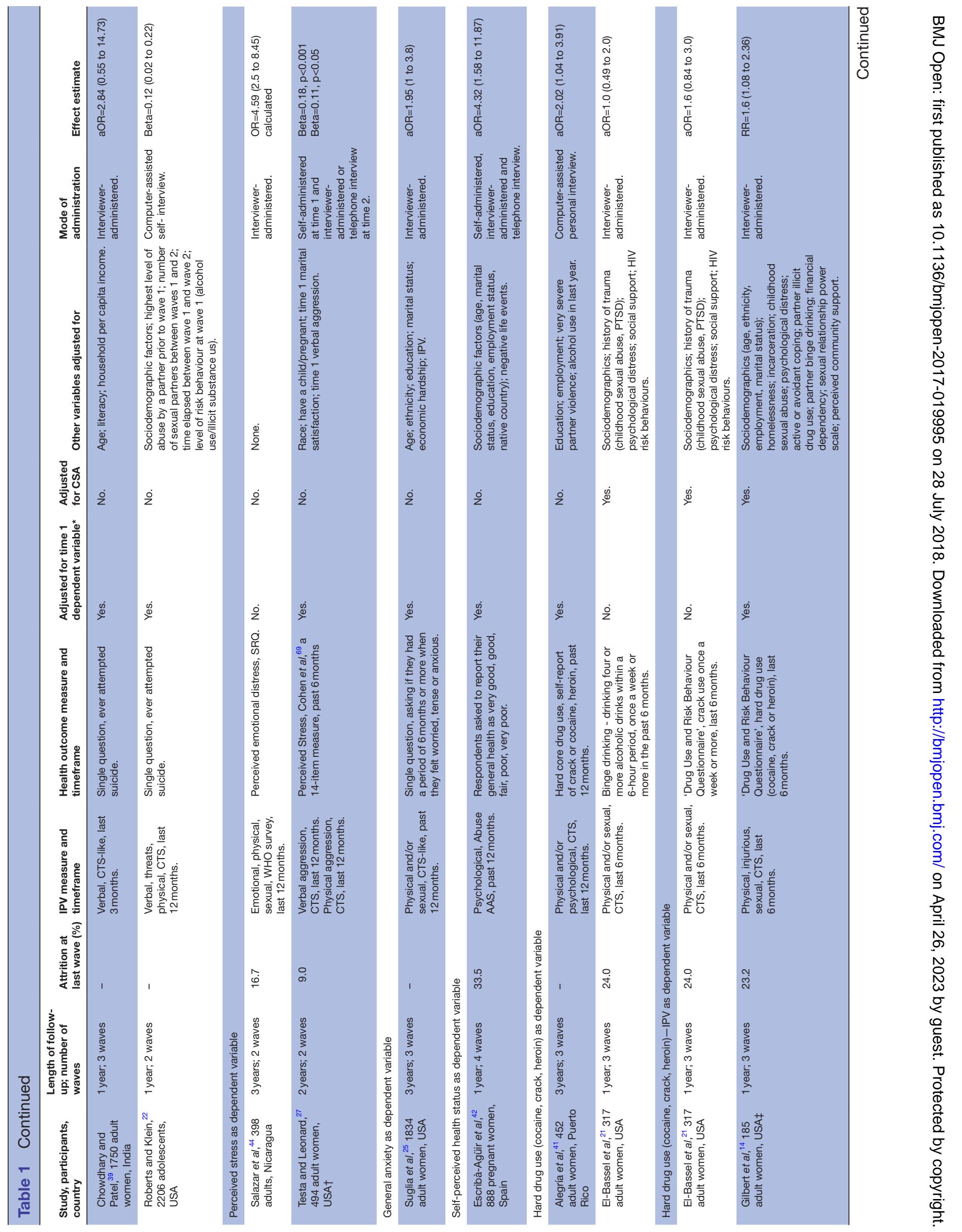




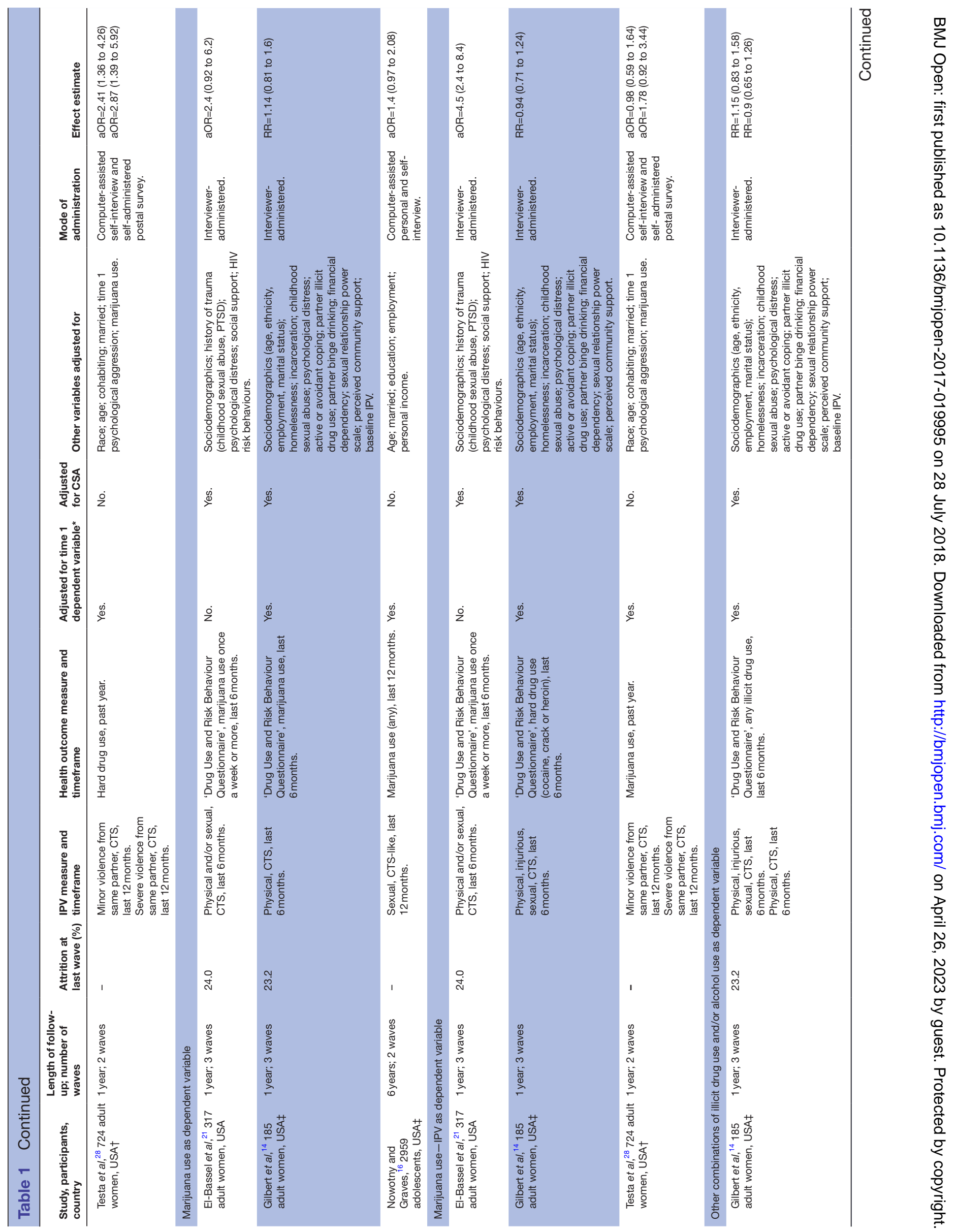




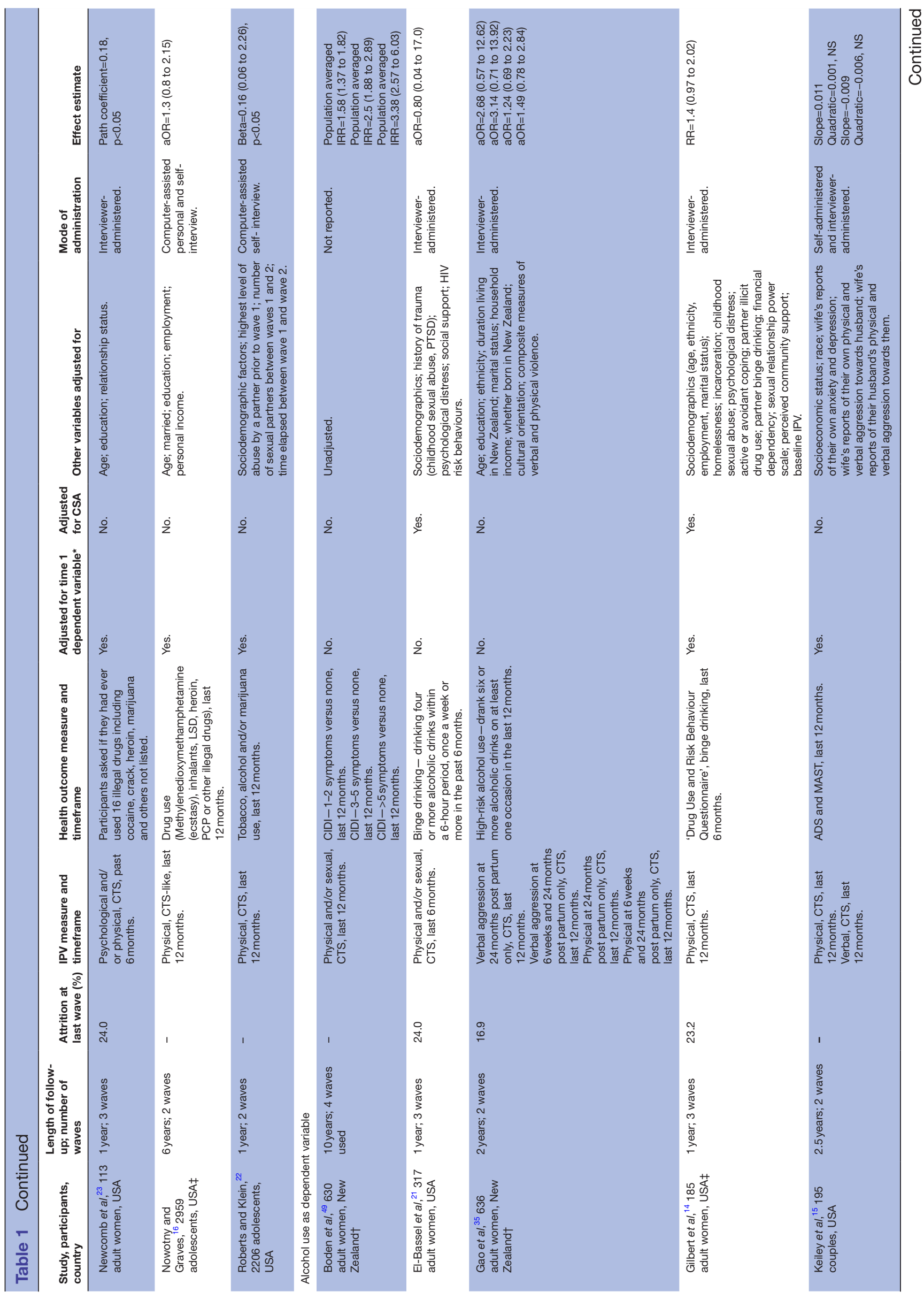

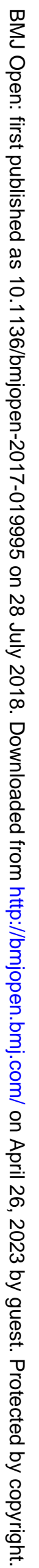




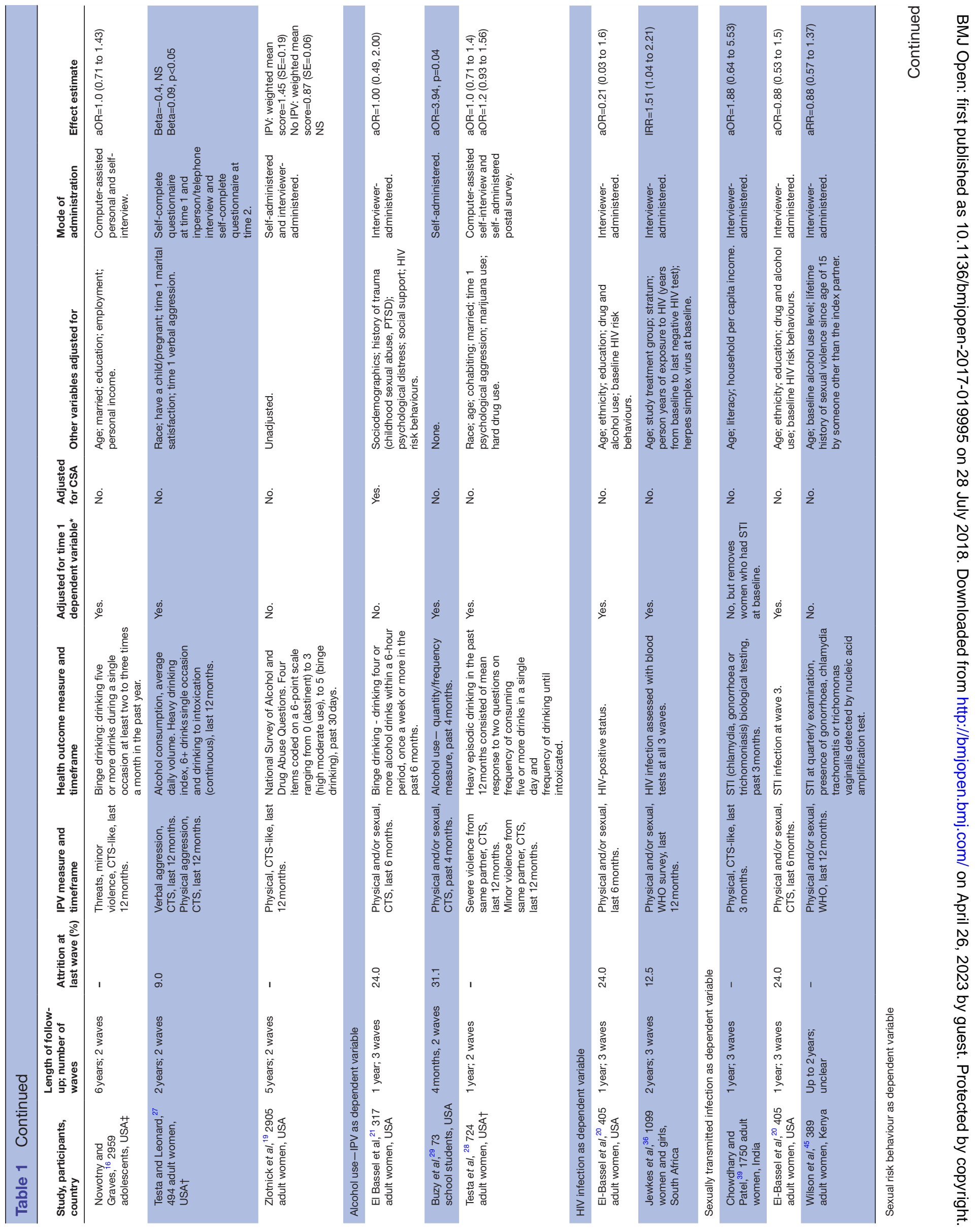



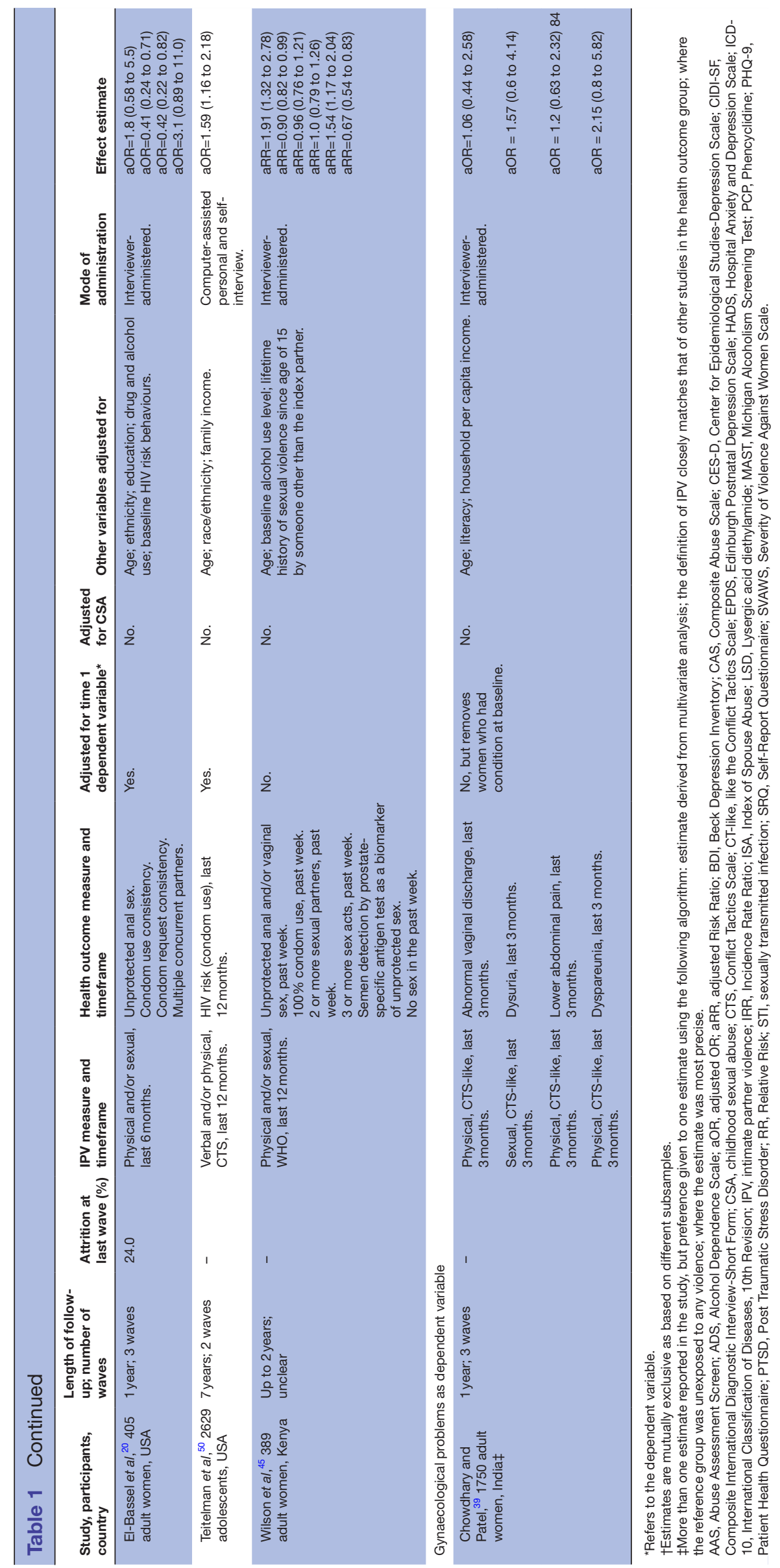

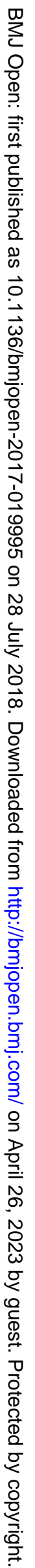




\section{Data analysis}

Analyses were conducted by LJB and KD using Stata V.14.0. Study characteristics and quality are summarised descriptively. Studies reported a range of effect estimates (eg, ORs, relative risks and correlation coefficients). Adjusted ORs were extracted directly from the publications, with the exception of one unadjusted OR which was calculated for a study on perceived stress, which is not one of the health outcomes included in the meta-analysis. Studies measured IPV or health outcomes in heterogeneous ways; therefore, the results are summarised descriptively for each health outcome. Where there were at least two estimates, random-effects meta-analysis was used to calculate the pooled ORs representing associations between IPV occurring up to and including the last 12 months and various health outcomes. Higgins' ${ }^{2}$ statistic, which describes the percentage of variability in point estimates that is due to heterogeneity rather than sampling error, ${ }^{12}$ was calculated. Some studies reported multiple estimates using overlapping definitions of IPV on the same sample of participants. In order to avoid double counting participants in these studies, which can lead to falsely precise pooled estimates, preference was given to one estimate using the following algorithm implemented in the following sequence: (1) those derived from multivariate analysis, (2) where the definition of IPV closely matched that of the other studies in the meta-analysis, (3) where the reference group was unexposed to any violence and (4) where the estimate was most precise (ie, the smallest CI). This algorithm was applied to three studies. Studies that provided multiple estimates, but on different subsamples of participants were included in the meta-analysis. Studies that reported other types of estimate (eg, correlations coefficients, betas, risk ratios) are documented separately.

\section{Patient and public involvement}

Patients and the public were not involved in this systematic review.

\section{RESULTS}

\section{Study characteristics}

Thirty-five separate cohort studies described in 36 articles published between 2002 and 2017 with 48863 participants met the inclusion criteria and contained 174 effect estimates of association between IPV and health outcomes. Eighteen articles were from the $\mathrm{USA}^{13-30}$ three from Australia, ${ }^{31-33}$ two from New Zealand, ${ }^{34} 35$ three from South Africa, ${ }^{36-38}$ two from India, ${ }^{39}{ }^{40}$ one from Puerto Rico, ${ }^{41}$ one from Spain, ${ }^{42}$ one from Korea, ${ }^{43}$ one from Nicaragua, ${ }^{44}$ one from Kenya, ${ }^{45}$ one from Japan, ${ }^{46}$ one from the $\mathrm{UK}^{47}$ and one from Tanzania. ${ }^{48}$ Among the 35 cohort studies, 11 were household surveys, ${ }^{1319272832353943444749} 14$ sampled participants from clinical settings, ${ }^{4} 202123252630313340424546487$ from schools $^{16172224293650}$ and 3 from the local community. ${ }^{153841}$ Some studies were based on subpopulations of women, including one study (reported in two papers) of women receiving methadone maintenance treatment, ${ }^{20} 21$ women attending a clinic with depressive symptoms at baseline, ${ }^{31}$ HIV-positive female sex workers ${ }^{45}$ and eight studies of pregnant women. ${ }^{30} 3338404246-48$ Six studies focused on adolescents ${ }^{16-18222429}$ and one (reported in two papers) included women and young girls. ${ }^{36} 37$

Table 2 presents the different health outcomes measured in the studies, the number of studies that measure each health condition, the overall number of estimates that contribute to each health condition and the number of estimates that contribute to the meta-analysis.

Table 1 summarises quality issues in relation to the 36 papers reporting on 35 separate cohort studies included in the review. All but 3 of the 35 cohort studies used recognised, validated IPV instruments or used items that were taken from validated instruments. ${ }^{4043}$ All but nine studies assessed for IPV that occurred in the last 12 months; one measured IPV in the last 3 months, ${ }^{39}$ two in the last 6 months, ${ }^{20} 2123$ one in the last 4 months, ${ }^{29}$ four measured IPV that occurred during pregnancy, ${ }^{46-48}$ and one measured IPV during or within 12 months of pregnancy. ${ }^{30}$ Most of the studies assessed for physical and/or sexual violence from a partner, with some also including threats, emotional or verbal abuse. The attrition rate was reported or calculated in 19 studies and ranged from $4.6 \%{ }^{48}$ to $37.4 \% .^{31}$ The length of follow-up ranged from 1 month $^{46}$ to 10 years, ${ }^{49}$ and the number of waves ranged from two (multiple studies) to six. ${ }^{33}$ The smallest sample size was 73 adolescents ${ }^{29}$ and the largest was 1303 adult women. ${ }^{48}$ Table 1 presents all study estimates grouped by health outcome.

\section{IPV and depressive symptoms}

Thirteen studies examined the relationship between recent IPV and depressive symptoms, ${ }^{13} 1719$ 22-26 3132373943 of which one examined the association in both directions. ${ }^{43}$ Of these, nine studies provided nine estimates of association between IPV and subsequent depressive symptoms. 131922232531323943 Eight of these estimates showed a positive direction of association between experience of IPV and subsequent depressive symptoms. 913192223313239 Of the nine estimates of the association between IPV and subsequent depression, all but two reached statistical significance. ${ }^{25} 39$ Five studies provided five estimates of association between depression and subsequent IPV, all of which showed a positive and statistically significant relationship. 1724263743

We were able to include seven estimates reporting binary IPV measures and binary depressive symptoms or disorder measures in the meta-analysis. For IPV and subsequent depressive symptoms or disorder, the pooled OR from five estimates ${ }^{1325313239}$ was 1.76 (95\% CI 1.26 to $\left.2.44, \mathrm{I}^{2}=37.5 \%, \mathrm{p}=0.172\right)$. Two estimates ${ }^{1737}$ were included in the meta-analysis of the relationship between depressive symptoms and subsequent IPV, resulting in a pooled OR of 1.72 (95\% CI 1.28 to $\left.2.31, \mathrm{I}^{2}=0.0 \%, \mathrm{p}=0.752\right)$. One study, not included in the meta-analysis, examined the 
Table 2 Health outcomes/health risk behaviours measured in the 35 studies and the number of estimates

\begin{tabular}{|c|c|c|}
\hline Health outcome & Studies and estimates (n), references & $\begin{array}{l}\text { Estimates in the meta- } \\
\text { analysis (n) }\end{array}$ \\
\hline Depression & 13 studies $^{13} 171922-2631323739$ 43; 13 estimates & 7 \\
\hline Postpartum depression & 8 studies $^{30} 3338404246-48 ; 11$ estimates & 7 \\
\hline Suicide attempts & 2 studies $^{22}{ }^{39} ; 2$ estimates & NA \\
\hline Perceived stress & 2 studies $^{27} 44 ; 3$ estimates & NA \\
\hline General anxiety & 1 study $^{25} ; 1$ estimate & NA \\
\hline Self-perceived health status & 1 study $^{42} ; 1$ estimate & NA \\
\hline Hard drug use & 4 studies ${ }^{14212841} ; 6$ estimates & 5 \\
\hline Marijuana use & 4 studies $^{14162128} ; 7$ estimates & 5 \\
\hline Other combinations of illicit drug/alcohol use & 4 studies ${ }^{14} 162223 ; 5$ estimates & NA \\
\hline Alcohol use & 10 studies $^{14-16192127-293549} ; 18$ estimates & 9 \\
\hline HIV infection & 2 studies $^{20}{ }^{36} ; 3$ estimates & NA \\
\hline Sexually transmitted infections & 3 studies $^{20} 3945 ; 2$ estimates & NA \\
\hline Sexual risk behaviours & 3 studies $^{182045} ; 8$ estimates & NA \\
\hline Abnormal vaginal discharge & 1 study ${ }^{39} ; 3$ estimates & NA \\
\hline Dysuria & 1 study $^{39} ; 3$ estimates & NA \\
\hline Lower abdominal pain & 1 study ${ }^{39} ; 3$ estimates & NA \\
\hline Dyspareunia & 1 study ${ }^{39} ; 3$ estimates & NA \\
\hline
\end{tabular}

NA, not applicable as study estimates were continuous and could not be included in a meta-analysis.

bidirectional relationship between IPV and depression. ${ }^{43}$ A Korean study of married women found that IPV at wave 1 was positively associated with the depression level at wave 1 (beta $=0.030, \mathrm{SE}=0.03, \mathrm{p}<0.001$ ), but negatively associated with the growth rate of depression over the study period (beta $=-0.03, \mathrm{SE}=0.01, \mathrm{p}=0.004)$. IPV experienced at wave 4 was associated with a larger growth rate of depression in the model (beta $=3.34, \mathrm{SE}=0.61, \mathrm{p}<0.001$ ) and the experience of IPV at wave 1 (beta $=0.68, \mathrm{SE}=0.11$, $\mathrm{p}<0.001$ ) (see figure 2).

\section{Depression measurement}

Of the nine studies that measured IPV and subsequent depressive symptoms, one measured depressive symptoms occurring in the past 12 months, ${ }^{25}$ one in the last 2 weeks, ${ }^{31}$ five in the last week, ${ }^{13} 19223243$ one "current ${ }^{39}$ and one did not specify a time period. ${ }^{23}$ Of the five studies that measured depressive symptoms and subsequent IPV, three measured depressive symptoms in the past week, ${ }^{173743}$ one in the past 6 months $^{24}$ and one did not specify a time period. ${ }^{26}$

All but one of the studies used screening questionnaires that measured depressive symptoms as opposed to diagnostic tools. Of the 13 studies, eight used the Center for Epidemiological Studies-Depression Scale, ${ }^{13171922} 23323743$ one study used the WHO International Classification of Diseases, 10th Revision, ${ }^{39}$ one used the Composite International Diagnostic Interview-Short Form (CIDI-SF), ${ }^{25}$ one used the Patient Health Questionnaire, ${ }^{31}$ one used the Beck Depression Inventory $(\mathrm{BDI})^{26}$ and one used a scale from Kandel and Davies. ${ }^{24}$

\section{Common risk factors/confounding}

Of the nine studies that measured IPV and subsequent depressive symptoms and disorder, all but one controlled for time 1 levels of depression. Chowdhary and Patel ${ }^{39}$ excluded women with baseline depressive disorder in their analysis, but this may have resulted in the exclusion of cases of IPV that preceded depressive symptoms at baseline and the remaining cases may not have been representative of women experiencing IPV. All but one of the five studies that measured depressive symptoms and later IPV controlled for time 1 levels of IPV ${ }^{26}$ Of the 13 studies, all but 2 controlled for sociodemographic factors. ${ }^{26}{ }^{39}$ Other confounders were not comprehensively controlled for. Two studies controlled for childhood physical and/or sexual abuse ${ }^{1731}$ and two for alcohol use, ${ }^{2437}$ of which one also controlled for childhood adversity which measured emotional and physical neglect, and physical and sexual abuse. ${ }^{37}$ There were no discernible differences in effect estimates regardless of which confounders were adjusted for, and studies found similar directions and varying magnitudes of association.

\section{IPV and postpartum depressive symptoms}

Eight studies provided eight estimates of association between IPV and subsequent postpartum depressive symptoms. ${ }^{30} 3338404246-48$ All eight estimates showed a positive direction of association between IPV and subsequent postpartum depressive symptoms, with all but one of the estimates reaching statistical significance. ${ }^{46}$ Seven estimates from five studies were included in the meta-analysis of the relationship between IPV and 


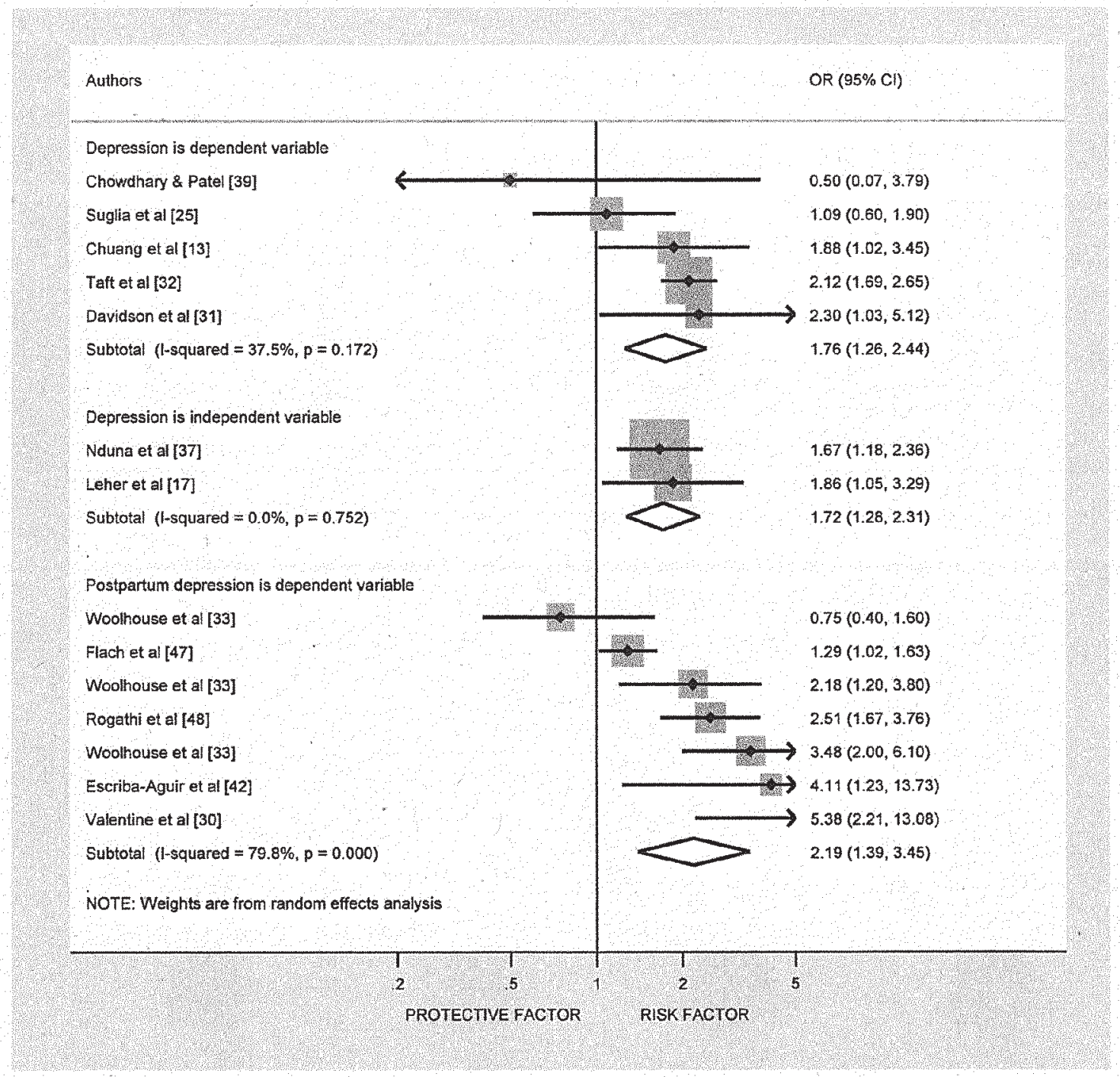

Notes: Woolhouse estimates are based on different sub-samples and are mutually exclusive.

Meta-analysis with depression as the dependent variable was also undertaken excluding the Chowdhary study,

but it did not materially change the overall pooled estimate $\left(\mathrm{OR}=1.83 ; 95 \% \mathrm{CI} 1.35-2.49 ; I^{2}=35.1 \%, \mathrm{p}=0.202\right)$

Figure 2 Forest plot estimates of the association between intimate partner violence and depression. Woolhouse et $a^{33}$ estimates are based on different subsamples and are mutually exclusive. Meta-analysis with depression as the dependent variable was also undertaken excluding the Chowdhary and Patel ${ }^{39}$ study which removes women with baseline depressive disorder from the analysis, but it did not materially change the overall pooled estimate $(\mathrm{OR}=1.85,95 \% \mathrm{Cl} 1.35$ to $\left.2.49, \mathrm{I}^{2}=35.1 \%, \mathrm{p}=0.202\right)$.

subsequent postpartum depression, ${ }^{3033424748}$ resulting in a pooled OR of 2.19 (95\% CI 1.39 to 3.45$)$. This was heterogeneous $\left(\mathrm{I}^{2}=79.8 \%, \mathrm{p}=0.000\right)$. One of the studies examined the bidirectional relationship and found that depression symptom severity was associated with a greater risk of subsequent IPV. ${ }^{38}$ Each 5-point difference in the Edinburgh Postnatal Depression Scale (EPDS) was associated with a 0.9-point to 2.3-point difference in subsequent IPV risk (beta $=0.054,95 \%$ CI 0.030 to 0.079$)$.

\section{Postpartum depression measurement}

Of the eight studies that measured IPV and subsequent depressive symptoms, one measured depressive symptoms occurring in the past 12 months, ${ }^{42}$ six studies measured depressive symptoms in the last week ${ }^{33} 384046-48$ and one study did not specify the time period. ${ }^{30}$ One study measured postpartum depression in the last week and subsequent IPV. $^{38}$ Of the eight studies, six used the EPDS, 333840424748 one used the Hospital Anxiety and Depression Scale ${ }^{46}$ and one used the BDI. ${ }^{30}$

\section{Common risk factors/confounding}

Six of the eight studies that examined IPV and subsequent postpartum depression controlled for time 1 levels of depressive symptoms. ${ }^{30} 3338424647$ One study did not control for time 1 levels of depressive symptoms as it was not significant in the bivariate analysis. ${ }^{48}$ Five studies 
controlled for sociodemographic factors. ${ }^{33} 38424648$ One study controlled for HIV serostatus ${ }^{38}$ and one controlled for HIV/AIDS diagnosis. ${ }^{48}$

\section{IPV and alcohol use}

Ten studies examined the relationship between recent IPV and alcohol use. Of these, eight studies provided 15 estimates of association between IPV and subsequent alcohol use. $^{14-161921 ~ 27-293549}$ All but one of these 15 estimates showed a positive direction of association between IPV and subsequent alcohol use,${ }^{15}$ with two studies providing 4 estimates which reached statistical significance. ${ }^{27} 49$ Three studies $^{21} 2829$ provided three estimates showing a positive direction of association between alcohol use and subsequent IPV, of which one was statistically significant. ${ }^{29}$

For IPV and later alcohol use, the pooled OR from six estimates provided by three studies ${ }^{162135}$ was $1.19(95 \%$ CI 0.91 to $1.55, \mathrm{I}^{2}=0.0 \%, \mathrm{p}=0.523$ ). Three estimates from two studies ${ }^{2128}$ were included in the meta-analysis of the relationship between alcohol use and subsequent IPV, resulting in a pooled OR of 1.11 (95\% CI 0.91 to 1.35 , $\mathrm{I}^{2}=0.0 \%, \mathrm{p}=0.672$ ).

\section{Alcohol use measurement}

Of the eight studies that measured IPV and subsequent alcohol use, five measured alcohol use in the last 12 months, ${ }^{1516273549}$ two in the last 6 months $^{1421}$ and one in the last 30 days. ${ }^{19}$ Of the three studies that measured alcohol use and subsequent IPV, one measured alcohol user in the last 12 months, ${ }^{28}$ one in the last 4 months ${ }^{29}$ and one in the last 6 months. ${ }^{21}$ Alcohol consumption was measured in a variety of ways. Of the 10 studies, one assessed alcohol abuse or dependence using the CIDI-SF, ${ }^{49}$ four measured binge drinking which was based on the number of alcoholic drinks consumed on one occasion, ${ }^{14} 162135$ three measured heavy drinking which was assessed using a combined quantity-frequency measure, ${ }^{27-29}$ one used the Alcohol Dependence Scale and the Michigan Alcohol Screening Test, ${ }^{15}$ and one used the National Survey of Alcohol and Drug Abuse. ${ }^{19}$

\section{Common risk factors/confounding}

Of the eight studies that measured IPV and subsequent alcohol use, only four adjusted for time 1 levels of alcohol use. $^{14-1627}$ Of the three studies that examined the association between alcohol use and subsequent IPV, all but one adjusted for time 1 levels of IPV. ${ }^{21}$ Of the 10 studies, 7 controlled for sociodemographic factors. ${ }^{14-1621272835}$ Two studies adjusted for a history of trauma. El-Bassel $e t a l^{21}$ controlled for childhood sexual abuse, post-traumatic stress disorder, multiple concurrent partners and frequency of condom use. Gilbert $e t \mathrm{al}^{14}$ also controlled for childhood sexual abuse, as well as psychological distress, coping strategies, the partner's illicit drug use and binge drinking and sexual relationship power. Regardless of the confounders controlled for, all but one study found a positive direction of association and reported varying magnitudes of association. ${ }^{27}$

\section{IPV and hard drug use (crack, cocaine, heroin)}

Four studies examined the relationship between recent IPV and hard drug use, ${ }^{1421} 2841$ of which one reported an association in both directions. ${ }^{21}$ Two studies provided two estimates of IPV and subsequent hard drug use, both of which showed a positive direction of association, although only one was statistically significant. ${ }^{21}{ }^{41}$ The pooled OR from these studies was 2.05 (95\% CI 1.19 to $3.52, \mathrm{I}^{2}=0.0 \%$, $\mathrm{p}=0.948$ ). Three studies provided four estimates of hard drug use and subsequent IPV, which showed a positive direction of association and three of these were statistically significant. ${ }^{142128}$ Three of these estimates were included in the meta-analysis, resulting in a pooled OR of 2.20 (95\% CI 1.52 to $\left.3.17, \mathrm{I}^{2}=0.0 \%, \mathrm{p}=0.455\right)$.

\section{Hard drug use measurement}

Of the two studies that measured IPV and subsequent hard drug use, one study measured drug use in the last 12 months ${ }^{41}$ and the other in the last 6 months. ${ }^{21}$ Of the three studies that measured hard drug use and subsequent IPV, one assessed use in the last 12 months $^{28}$ and two in the last 6 months. ${ }^{1421}$ Of the four studies, two used the Drug Use and Risk Behaviour Questionnaire ${ }^{1421}$ and two asked about use of specific hard drugs including crack, cocaine and heroin. ${ }^{28}{ }^{41}$ Of the latter, one of the studies used two methods for assessing hard drug use at each wave, including self-report information only and combined self-report and toxicological information. ${ }^{41}$

\section{Common risk factors/confounding}

Of the two studies that measured IPV and subsequent hard drug use, one controlled for time 1 levels of hard drug use. ${ }^{41}$ Of the three studies that measured hard drug use and subsequent IPV, two controlled for time 1 levels of IPV. ${ }^{1428}$ All four studies controlled for sociodemographic factors. El-Bassel $e t a l^{21}$ controlled for childhood sexual abuse, post-traumatic stress disorder, multiple concurrent partners and frequency of condom use. Gilbert et $a l^{14}$ controlled for childhood sexual abuse, psychological distress, coping strategies, the partner's illicit drug use and binge drinking and sexual relationship power.

\section{IPV and marijuana use}

Four studies examined the relationship between recent IPV and marijuana use, ${ }^{14162128}$ of which two studies reported an association in both directions. ${ }^{14}{ }^{21}$ Three studies provided three estimates of IPV and subsequent marijuana use, all showing a positive direction of association, although none were statistically significant. ${ }^{141621}$ Two of these studies were included in the meta-analysis, resulting in a pooled OR of 1.52 (95\% CI 1.04 to $\left.2.24, \mathrm{I}^{2}=5.4 \%, \mathrm{p}=0.304\right)$. Three studies provided four estimates of marijuana use and subsequent IPV, ${ }^{142128}$ of which one showed a positive and statistically significant relationship. Three of these estimates were included in the meta-analysis, resulting in a pooled OR of $1.96(95 \%$ CI 0.8 to 4.83$)$. This was heterogeneous $\left(\mathrm{I}^{2}=85.4 \%\right.$, $\mathrm{p}=0.001$ ). 
Marijuana use measurement

Of the four studies, two measured marijuana use in the last 12 months ${ }^{1628}$ and two in the last 6 months. ${ }^{1421}$ All studies used self-report information to assess for marijuana use.

\section{Common risk factors/confounding}

Of the three studies that measured IPV and subsequent marijuana use, two controlled for time levels of marijuana use. $^{14} 16$ Of the three studies that measured marijuana use and subsequent IPV, two controlled for time levels of IPV. ${ }^{142}$ All the studies controlled for sociodemographic factors. El-Bassel $e t a l^{21}$ controlled for childhood sexual abuse, post-traumatic stress disorder, multiple concurrent partners and frequency of condom use. Gilbert et $a l^{14}$ controlled for childhood sexual abuse, psychological distress, coping strategies, the partner's illicit drug use and binge drinking and sexual relationship power.

\section{IPV and STIs (excluding HIV)}

Three studies provided three estimates of the association between recent IPV and subsequent sexually transmitted infections (STIs), ${ }^{20} 3945$ of which one showed a positive and statistically significant relationship. ${ }^{39}$ The meta-analysis of two of these studies ${ }^{20}{ }^{39}$ resulted in a pooled OR of $1.10\left(95 \%\right.$ CI 0.56 to $\left.2.18, \mathrm{I}^{2}=35.5 \%, \mathrm{p}=0.214\right)$.

\section{STI measurement}

One study assessed for STIs (chlamydia, gonorrhoea or trichomoniasis) within the last 3 months using biological measures, ${ }^{39}$ another relied on self-report to assess for STIs at the last wave, ${ }^{20}$ and the third study assessed women quarterly for gonorrhoea, chlamydia or trichomoniasis using biological measures. ${ }^{45}$

\section{Common risk factors/confounding}

All the studies controlled for sociodemographic factors. El-Bassel $e t$ al's study ${ }^{21}$ of women attending a methadone maintenance clinic adjusted for time 1 HIV risk factors (ie, frequency of condom use, frequency of requesting condom use, having unprotected anal sex, exchanging sex for drugs, being HIV-positive and having had an STI), as well as drug and alcohol use. Chowdhary and Patel ${ }^{39}$ removed women with an STI at time 1 from the analysis. However, this would likely have introduced bias in the resulting cases, as it would have excluded women with IPV that preceded the acquisition of an STI at baseline. Wilson et $a r_{\mathrm{s}}{ }^{45}$ study of HIV-positive sex workers did not control for time 1 sexual risk behaviours, although it did control for a lifetime history of sexual violence since the age of 15 by someone other than the index partner. Figure 3 presents the forest plots for alcohol use, hard drug use, marijuana use and STIs.

\section{DISCUSSION}

\section{Summary of main findings}

Our review identified cohort studies that examined the relationship between recent IPV (ie, IPV occurring up to and including the last 12 months) and depression, postpartum depression, alcohol use, hard drug use, marijuana use and STIs. Although a few other health or health-related outcomes were identified (ie, sexual risk behaviours, HIV infection, general anxiety, perceived stress and gynaecological problems), these could not be included in a meta-analysis because there was only one estimate. We found evidence consistent with a bidirectional relationship between recent IPV and depressive symptoms. Recent IPV was also associated with increased symptoms of postpartum depression. There was some evidence of a bidirectional relationship between recent IPV and hard drug use, and IPV and subsequent marijuana use, although there were a limited number of studies. There was no evidence of an association between recent IPV and alcohol or STIs, although the evidence was weak with few studies and inconsistent measurement of alcohol and STIs.

Although the search strategy did not limit the types of health outcomes identified, the review found no cohort studies for recent IPV exposure and non-communicable diseases such as cardiovascular disease, hypertension and obesity. Nor did we find longitudinal evidence for recent experience of IPV and post-traumatic stress disorder or anxiety disorder. There is limited evidence from cross-sectional data that lifetime IPV increases the risk of cardiovascular disease. ${ }^{51}$ Cohort studies measuring history of IPV have reported an association with increased body mass index ${ }^{52}$ increased risk for cardiovascular disease ${ }^{53}$ and hypertension. ${ }^{54}$ Physiological mechanisms may explain the association between IPV and some adverse health outcomes through complex neural, neuroendocrine and immune responses to acute and chronic stress. For example, sustained and acute elevated stress levels have been linked to cardiovascular disease, hypertension, gastrointestinal disorders and chronic pain. When exposed to prolonged or acute stress, areas of the brain (eg, hippocampus, amygdala and prefrontal cortex) undergo structural changes that can impact on mental and cognitive functioning, which can lead to mental disorders. ${ }^{55}$

We found evidence consistent with a bidirectional relationship between recent experience of IPV and depressive symptoms. The magnitude of the association in either direction is similar to that reported in our previous review of 'ever' IPV and depressive symptoms, ${ }^{4}$ although there were fewer estimates in our meta-analysis of recent IPV and depressive symptoms.

All the studies on postpartum depressive symptoms conceptualised IPV as the dependent variable and there was evidence that recent experience of IPV or IPV during pregnancy increased symptoms of subsequent postpartum depression, although there was substantial heterogeneity. The magnitude of the association was slightly lower ( $\mathrm{OR}=2.19,95 \% \mathrm{CI} 1.39$ to 3.45$)$ compared with Howard $e t a l,{ }^{56}$ who reported a threefold increase in the levels of depressive symptoms in the postnatal period after having experienced IPV during pregnancy $(\mathrm{OR}=3.1$, 
Authors

OR $(95 \% \mathrm{Cl})$

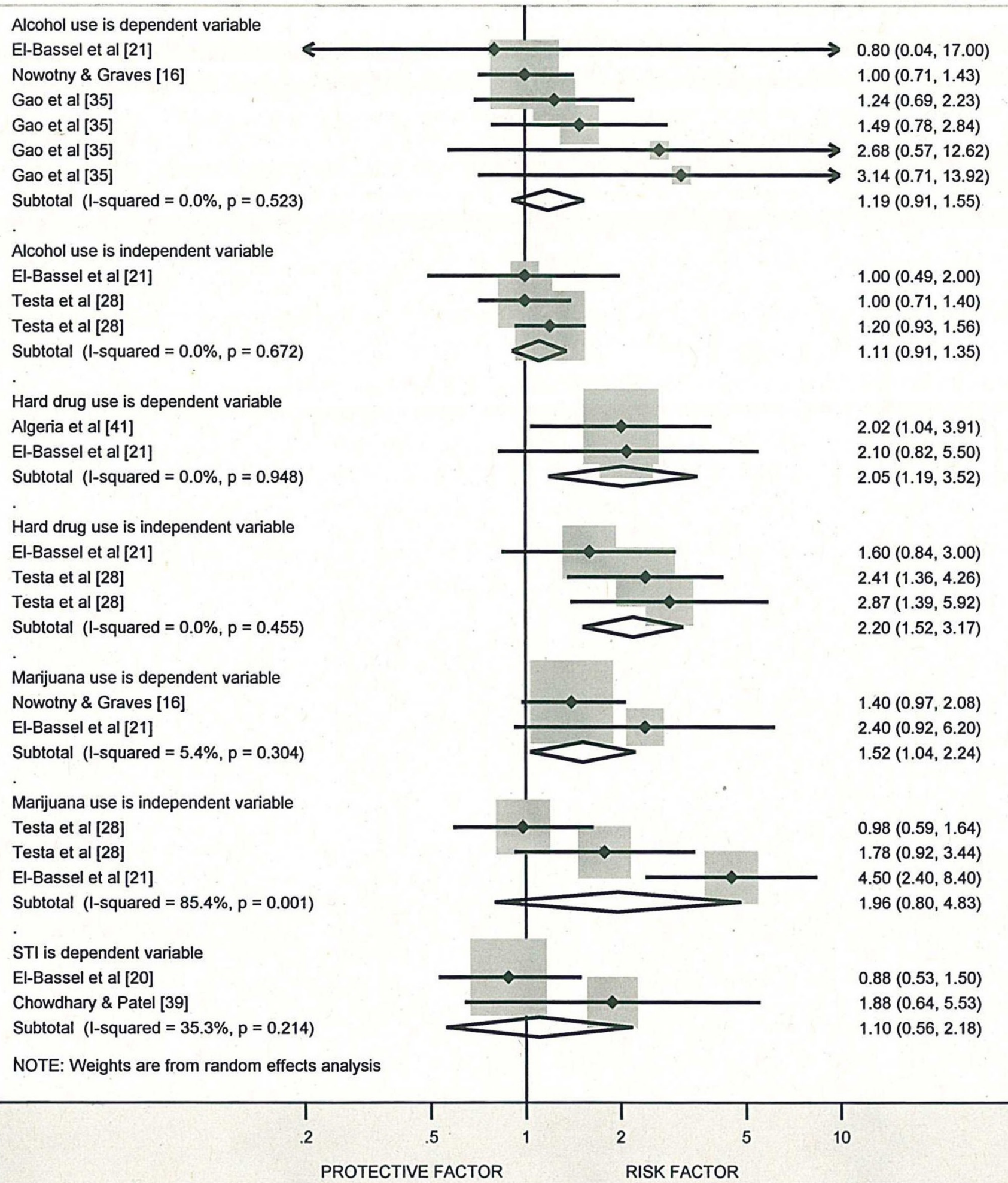

Figure 3 Forest plot estimates of the association between intimate partner violence and alcohol use, hard drug use, marijuana use and sexually transmitted infections (STIs). Estimates from Gao et $\left.a\right|^{35}$ and Testa et $a^{28}$ are based on different subsamples and are mutually exclusive.

95\% CI 2.7 to 3.6). However, the authors state that study heterogeneity and lack of data on baseline symptoms prevented conclusions on temporality. In addition, we excluded one study that was included in the Howard et $a l^{56}$ review as it measures postnatal depressive symptoms using the EPDS at the final wave, but assesses common 
mental health disorders during pregnancy with the Self-Reporting Questionnaire-20. ${ }^{57}$ A recently published systematic review explored studies of IPV during pregnancy and perinatal mental disorders in low-income and middle income countries. However, most of the studies were cross-sectional and considered partner violence experienced during pregnancy. Furthermore, estimates were not pooled in a meta-analysis. ${ }^{58}$

There was no evidence of an association between recent IPV and alcohol use in either direction. This might be because there were fewer estimates in the meta-analysis of recent IPV, and measurement of problematic alcohol use was conceptualised in a number of different ways, for example, binge drinking, heavy episodic drinking and high-risk alcohol use, which may have diluted the effect. None of the estimates in the meta-analysis measured alcohol use disorder. Furthermore, not all the estimates in the meta-analysis controlled for time 1 levels of alcohol use, and only one included the perpetrator's alcohol use, which may be related to IPV and/or the woman's drinking behaviour. This finding is in contrast to our previous review of 'ever' IPV and alcohol use, which did find evidence consistent with a bidirectional relationship. ${ }^{5}$ Although the pooled estimates in both reviews are based on studies that assess binge drinking, the Devries et a $\tilde{e}$ review includes estimates of IPV that occurred in the distant past (ie, before the last 12 months).

Our review found evidence consistent with a bidirectional relationship between recent IPV and hard drug use. However, this finding should be treated with caution as there were very few studies overall, and one of the studies was based on a sample of women attending a methadone maintenance clinic. For marijuana use, there were few studies, but the evidence suggests that IPV predicts subsequent marijuana use. Pooled estimates did not support that marijuana use predicts subsequent IPV, although estimates were heterogeneous. The evidence for recent IPV and STI infection was in conflicting directions and there were only two estimates. Our review adds to previous systematic reviews as it focuses on longitudinal studies that measure recent experiences of IPV. Furthermore it includes a broader range of health or health-related outcomes and explores bidirectionality. The review also highlights that longitudinal studies on recent IPV are lacking for important health outcomes that are known to be associated with partner violence.

\section{Limitations of the review}

To our knowledge, this is the first systematic review of cohort studies to measure the magnitude of the association between recent exposure to IPV and health outcomes/health risk behaviours. Although we conducted an extensive search of the global literature, the review has a number of limitations. Due to the large number of abstracts retrieved and the limited timeframe for the review, we were not able to employ double screening of abstracts. Citation tracking was not undertaken, although we conducted reference list screening of key systematic review papers. However, two researchers reviewed the final set of included papers, with a third reviewer of all full text papers where there was uncertainty about their inclusion. One researcher was responsible for extracting data from included papers. As some studies measured the outcome variable (either IPV or the health condition) continuously, it was not possible to combine all measures of effect, which limited the number of studies in the meta-analysis. However, we comment on the direction of the association of studies that were not included in the meta-analysis in the Results section for each health condition. It was not possible to quantitatively assess publication bias as too few studies were in the meta-analysis of each health condition.

\section{Sources of bias and limitations of included studies}

One of the main limitations of the included studies relates to the lack of consistency in controlling for key potential confounders. With regard to studies on depression, hard drug use and marijuana use, most controlled for time 1 levels of the health condition or IPV (where IPV was the dependent variable). Far fewer of the estimates on IPV and later alcohol use and IPV and STI controlled for time 1 levels of the health outcome.

With regard to the studies on depressive symptoms, only two controlled for early childhood trauma (ie, childhood sexual and/or physical abuse) and two controlled for alcohol use, even though both are known to increase the risk for depression. ${ }^{5960}$ This makes it difficult to rule them out as potential contributors to the causation of the outcomes. Nevertheless, we found that studies showed a positive direction of association, regardless of which variables were adjusted for, and there was no clear pattern of differing magnitude of association that indicated the relationship between IPV and depressive symptoms was not likely to be entirely accounted for by shared risk factors.

Little is known about the potential causal mechanisms between depression and subsequent IPV. However, women who are depressed may experience symptoms (eg, lethargy and withdrawal) that impact their capacity for engaging in self-care behaviours including helpseeking and contact with healthcare providers that could enable them to extricate themselves from the relationship. It is also plausible that earlier, perhaps unmeasured experiences of violence, such as childhood sexual abuse and trauma, are causing depression and later IPV, or that depression is mediating the relationship between childhood sexual abuse and later IPV. A path analysis with cross-sectional data supports this hypothesis, ${ }^{60}$ but few longitudinal studies have explored these relationships.

Only two studies on alcohol use controlled for childhood sexual abuse and one controlled for the partner's level of alcohol use, both of which are potential causes of women's alcohol use. It has been suggested that women who drink heavily are more likely to have a partner who drinks heavily, which can increase their risk of IPV because heavy alcohol use by men is associated with IPV perpetration. ${ }^{61}$ This can occur because people tend to choose a 
partner with similar drinking patterns to themselves or through the influence of their partner's drinking patterns and expectations. ${ }^{62}$ Research also suggests that the partner's or the woman's drinking may fuel conflict in the relationship. A nationally representative study from the USA found that couples with similar drinking patterns (eg, both abstinent or both binge drinkers) were less likely to experience IPV in their relationship compared with those with discordant drinking habits. ${ }^{63}$ This implies that relationship conflict may result in IPV, as opposed to alcohol use alone because high alcohol use would be more predictive than discordant use. Alcohol use was measured in a variety of ways, with most assessing binge drinking or heavy drinking and only two studies measuring alcohol dependence. Although heavy alcohol consumption increases the risk for disease, injury and premature death, ${ }^{6465}$ the adverse consequences may vary considerably between people who sporadically drink heavily and those who develop an alcohol use disorder. Although the evidence points to a bidirectional relationship between IPV and hard drug use and IPV and marijuana use, there were few estimates. Women may self-medicate with alcohol, tobacco or drugs in an attempt to cope with the trauma and stress of living in an abusive relationship, which in themselves are important risk factors for poor health. However, alcohol or substance abuse by the abuser or the woman has also been identified as a trigger to violent episodes or a factor that contributes to more severe violence. ${ }^{66}$ The evidence for the association between recent IPV and STIs is uncertain.

It was not possible to examine whether the duration or severity of the violence influenced the relationship between IPV and health. Studies conceptualised violence as physical, sexual, verbal or emotional (or psychological), with most using a combination of types of violence. Only one study provided estimates of minor and severe violence. Studies reported the timeframe in which the violence occurred, but not the duration.

The majority of the studies were from high-income countries, most notably the USA, and only eight studies were from middle-income countries, where it is known that the prevalence of past-year IPV is higher. Six of the studies were of adolescents, again mostly in high-income countries, where these were likely to be in dating relationships with no cohabitation. One study included young girls and women. Experiences of IPV in adult and adolescent relationships may be qualitatively different, in that there is a lower likelihood of experiencing systematic and chronic violence in dating relationships. ${ }^{67}$ About a third of the studies were drawn from clinical settings, schools or were taken from subpopulations and therefore subject to bias (eg, HIV-positive sex workers, women with depressive symptoms and women on methadone maintenance). More population-based cohort studies are needed in order to generalise the findings. Most studies measured physical violence and some modelled exposure to physical and sexual and other forms of violence separately. However, other forms of violence (eg, emotional abuse, threats) may also be associated with some of the health outcomes. Most studies constructed the reference categories for IPV as binary opposites, meaning that some participants in the reference group may have been exposed to other forms of IPV that were not measured or modelled. This can bias the effect estimates towards the null and underestimate the magnitude of the association between recent IPV and health outcomes. Some studies included only women who were in a relationship for all waves of data collection. However, research shows that the prevalence of IPV is higher among women who are no longer with abuser compared with those currently in a relationship, ${ }^{68}$ and excluding these women may dilute the association between IPV and health outcomes.

\section{IMPLICATIONS}

The evidence on the association between exposure to IPV and mental and physical health outcomes has important implications for the delivery of interventions and services. IPV against women has received increasing attention from public health experts globally. ${ }^{2}$ The results of this review indicate that healthcare providers and specialist IPV organisations should be aware of the bidirectional relationship between recent IPV and depression. Women with depression may be at risk of IPV, including IPV that is ongoing, and services, particularly healthcare, should be trained to enquire about IPV experiences and respond and refer appropriately. Little is known about what pattern of exposure to IPV is more strongly associated with different health outcomes. In order to establish these connections, longitudinal studies of IPV and health are needed that distinguish recent violence with no history from recent violence that is part of ongoing abuse and historical violence that no longer occurs. Other factors that are known to mediate the relationship, such as the duration and severity of IPV, childhood physical and sexual abuse, poverty-related stress, and risk behaviours such as alcohol and substance abuse, should be carefully considered in analyses.

Contributors Conceived and designed the study: LJB, KD. Data collection: LJB. Analysed the data: LJB, MR, KD. Wrote the first draft of the manuscript: LJB. Contributed to the writing of the manuscript: LJB, CW, KD, MR. Agreed with manuscript results and conclusions: LJB, CW, KD, MR.

Funding The authors have not declared a specific grant for this research from any funding agency in the public, commercial or not-for-profit sectors.

Competing interests None declared.

Patient consent Not required.

Ethics approval All data used in this review were already in the public domain and ethical approval was not required.

Provenance and peer review Not commissioned; externally peer reviewed.

Data sharing statement № additional data available.

Open access This is an open access article distributed in accordance with the Creative Commons Attribution Non Commercial (CC BY-NC 4.0) license, which permits others to distribute, remix, adapt, build upon this work non-commercially, and license their derivative works on different terms, provided the original work is properly cited, appropriate credit is given, any changes made indicated, and the use is non-commercial. See: http://creativecommons.org/licenses/by-nc/4.0/. 


\section{REFERENCES}

1. Devries KM, Mak JY, García-Moreno C, et al. Global health. The global prevalence of intimate partner violence against women. Science 2013;340:1527-8.

2. World Health Organization. Global and regional estimates of violence against women: prevalence and health effects of intimate partner violence and non-partner sexual violence. Geneva, Switzerland: World Health Organization, 2013.

3. Mokdad AH, Forouzanfar MH, Daoud F, et al. Global burden of diseases, injuries, and risk factors for young people's health during 1990-2013: a systematic analysis for the Global Burden of Disease Study 2013. Lancet 2016;387:2383-401.

4. Devries KM, Mak JY, Bacchus LJ, et al. Intimate partner violence and incident depressive symptoms and suicide attempts: a systematic review of longitudinal studies. PLoS Med 2013;10:e1001439.

5. Devries KM, Child JC, Bacchus LJ, et al. Intimate partner violence victimization and alcohol consumption in women: a systematic review and meta-analysis. Addiction 2014;109:379-91.

6. Hindin MJ, Kishor S, Ansam DL. Intimate Partner Violence among Couples in 10 DHS Countries: Predictors and Health Outcomes: DHS Analytical studies. Calverton, Maryland, USA, 2008.

7. World Health Organization. WHO multi-country study on women's health and domestic violence against women: Geneva, Switzerland, 2005.

8. Abramsky T, Devries K, Kiss L, et al. Findings from the SASA! Study: a cluster randomized controlled trial to assess the impact of a community mobilization intervention to prevent violence against women and reduce HIV risk in Kampala, Uganda. BMC Med 2014;12:122.

9. Kim JC, Watts $\mathrm{CH}$, Hargreaves JR, et al. Understanding the impact of a microfinance-based intervention on women's empowerment and the reduction of intimate partner violence in South Africa. Am J Public Health 2007;97:1794-802.

10. Wagman JA, Gray RH, Campbell JC, et al. Effectiveness of an integrated intimate partner violence and HIV prevention intervention in Rakai, Uganda: analysis of an intervention in an existing cluster randomised cohort. Lancet Glob Health 2015;3:e23-e33.

11. Glymour MM, Weuve J, Berkman LF, et al. When is baseline adjustment useful in analyses of change? An example with education and cognitive change. Am J Epidemiol 2005;162:267-78.

12. Higgins JP, Thompson SG. Quantifying heterogeneity in a metaanalysis. Stat Med 2002;21:1539-58.

13. Chuang $\mathrm{CH}$, Cattoi AL, McCall-Hosenfeld JS, et al. Longitudinal association of intimate partner violence and depressive symptoms. Ment Health Fam Med 2012;9:107-14.

14. Gilbert L, El-Bassel N, Chang M, et al. Substance use and partner violence among urban women seeking emergency care. Psychol Addict Behav 2012;26:226-35.

15. Keiley MK, Keller PS, El-Sheikh M. Effects of physical and verbal aggression, depression, and anxiety on drinking behavior of married partners: a prospective and retrospective longitudinal examination. Aggress Behav 2009;35:296-312.

16. Nowotny KM, Graves JL. Substance use and intimate partne violence victimization among White, African American, and Latina women. J Interpers Violence 2013;28:3301-18.

17. Lehrer JA, Buka S, Gortmaker S, et al. Depressive symptomatology as a predictor of exposure to intimate partner violence among US female adolescents and young adults. Arch Pediatr Adolesc Med 2006;160:270-6.

18. Teitelman AM, Ratcliffe SJ, Dichter ME, et al. Recent and Past Intimate Partner Abuse and HIV Risk Among Young Women. Journal of Obstetric, Gynecologic \& Neonatal Nursing 2008;37:219-27.

19. Zlotnick C, Johnson DM, Kohn R, Violence IP. Intimate partner violence and long-term psychosocial functioning in a national sample of American women. J Interpers Violence 2006;21:262-75.

20. El-Bassel N, Gilbert L, Wu E, et al. HIV and intimate partner violence among methadone-maintained women in New York City. Soc Sci Med 2005;61:171-83.

21. El-Bassel N, Gilbert L, Wu E, et al. Relationship between drug abuse and intimate partner violence: a longitudinal study among women receiving methadone. Am J Public Health 2005;95:1493-4.

22. Roberts TA, Klein J. Intimate partner abuse and high-risk behavior in adolescents. Arch Pediatr Adolesc Med 2003;157:375-80.

23. Newcomb MD, Carmona JV, Vargas Carmona J. Adult trauma and HIV status among Latinas: effects upon psychological adjustment and substance use. AIDS Behav 2004;8:417-28.

24. Foshee VA, Benefield TS, Ennett ST, et al. Longitudinal predictors of serious physical and sexual dating violence victimization during adolescence. Prev Med 2004;39:1007-16.
25. Suglia SF, Duarte CS, Sandel MT. Housing quality, housing instability, and maternal mental health. J Urban Health 2011;88:1105-16.

26. Levendosky AA, Bogat GA, Huth-Bocks AC, et al. The effects of domestic violence on the stability of attachment from infancy to preschool. J Clin Child Adolesc Psychol 2011;40:398-410.

27. Testa M, Leonard KE. The impact of marital aggression on women's psychological and marital functioning in a newlywed sample. J Fam Violence 2001;16:115-30.

28. Testa M, Livingston JA, Leonard KE. Women's substance use and experiences of intimate partner violence: a longitudinal investigation among a community sample. Addict Behav 2003;28:1649-64.

29. Buzy WM, McDonald R, Jouriles EN, et al. Adolescent Girls' Alcohol Use as a Risk Factor for Relationship Violence. J Res Adolesc 2004;14:449-70

30. Valentine JM, Rodriguez MA, Lapeyrouse LM, et al. Recent intimate partner violence as a prenatal predictor of maternal depression in the first year postpartum among Latinas. Arch Womens Ment Health 2011:14:135-43.

31. Davidson SK, Dowrick CF, Gunn JM. Impact of functional and structural social relationships on two year depression outcomes: A multivariate analysis. J Affect Disord 2016;193:274-81.

32. Taft AJ, Watson LF. Depression and termination of pregnancy (induced abortion) in a national cohort of young Australian women: the confounding effect of women's experience of violence. BMC Public Health 2008;8:75

33. Woolhouse H, Gartland D, Mensah F, et al. Maternal depression from early pregnancy to 4 years postpartum in a prospective pregnancy cohort study: implications for primary health care. BJOG 2015;122:312-21.

34. Boden JM, Fergusson DM, Horwood LJ. Alcohol misuse and relationship breakdown: findings from a longitudinal birth cohort Drug Alcohol Depend 2013;133:115-20.

35. Gao W, Paterson J, Abbott M, et al. Impact of current and past intimate partner violence on maternal mental health and behaviour at 2 years after childbirth: evidence from the Pacific Islands Families Study. Aust N Z J Psychiatry 2010;44:174-82.

36. Jewkes RK, Dunkle K, Nduna M, et al. Intimate partner violence, relationship power inequity, and incidence of HIV infection in young women in South Africa: a cohort study. Lancet 2010;376:41-8.

37. Nduna M, Jewkes RK, Dunkle KL, et al. Associations between depressive symptoms, sexual behaviour and relationship characteristics: a prospective cohort study of young women and men in the Eastern Cape, South Africa. J Int AIDS Soc 2010;13:44.

38. Tsai AC, Tomlinson M, Comulada WS, et al. Intimate Partner Violence and Depression Symptom Severity among South African Women during Pregnancy and Postpartum: Population-Based Prospective Cohort Study. PLoS Med 2016;13:e1001943.

39. Chowdhary N, Patel V. The effect of spousal violence on women's health: findings from the Stree Arogya Shodh in Goa, India. $J$ Postgrad Med 2008;54:306-12.

40. Patel V, Rodrigues M, DeSouza N, Gender DN. Gender, poverty, and postnatal depression: a study of mothers in Goa, India. Am $J$ Psychiatry 2002;159:43-7.

41. Alegría M, Vera M, Shrout $P$, et al. Understanding hard-core drug use among urban Puerto Rican women in high-risk neighborhoods. Addict Behav 2004;29:643-64.

42. Escribà-Agüir V, Royo-Marqués M, Artazcoz L, et al. Longitudinal study of depression and health status in pregnant women: incidence, course and predictive factors. Eur Arch Psychiatry Clin Neurosci 2013;263:143-51.

43. Kim J, Lee J. Prospective study on the reciprocal relationship between intimate partner violence and depression among women in Korea. Soc Sci Med 2013;99:42-8.

44. Salazar M, Valladares E, Ohman A, et al. Ending intimate partner violence after pregnancy: findings from a community-based longitudinal study in Nicaragua. BMC Public Health 2009;9:350.

45. Wilson KS, Deya R, Yuhas K, et al. A Prospective Cohort Study of Intimate Partner Violence and Unprotected Sex in HIVPositive Female Sex Workers in Mombasa, Kenya. AIDS Behav 2016;20:2054-64.

46. Kita S, Haruna M, Matsuzaki M, et al. Associations between intimate partner violence (IPV) during pregnancy, mother-to-infant bonding failure, and postnatal depressive symptoms. Arch Womens Ment Health 2016;19:623-34.

47. Flach C, Leese M, Heron J, et al. Antenatal domestic violence, maternal mental health and subsequent child behaviour: a cohort study. BJOG 2011;118:1383-91.

48. Rogathi JJ, Manongi R, Mushi D, et al. Postpartum depression among women who have experienced intimate partner violence: 
A prospective cohort study at Moshi, Tanzania. J Affect Disord 2017;218:238-45.

49. Boden JM, Fergusson DM, Horwood LJ. Alcohol misuse and violent behavior: findings from a 30-year longitudinal study. Drug Alcohol Depend 2012;122:135-41.

50. Teitelman AM, Ratcliffe SJ, Dichter ME, et al. Recent and past intimate partner abuse and HIV risk among young women. $J$ Obstet Gynecol Neonatal Nurs 2008;37:219-27.

51. Stene LE, Jacobsen GW, Dyb G, et al. Intimate partner violence and cardiovascular risk in women: a population-based cohort study. $J$ Womens Health 2013;22:250-8.

52. Clark CJ, Spencer RA, Everson-Rose SA, et al. Dating violence, childhood maltreatment, and BMI from adolescence to young adulthood. Pediatrics 2014;134:678-85.

53. Clark CJ, Alonso A, Everson-Rose SA, et al. Intimate partner violence in late adolescence and young adulthood and subsequent cardiovascular risk in adulthood. Prev Med 2016;87:132-7.

54. Clark CJ, Everson-Rose SA, Alonso A, et al. Effect of partner violence in adolescence and young adulthood on blood pressure and incident hypertension. PLoS One 2014;9:e92204.

55. Miller AH. Neuroendocrine and immune system interactions in stress and depression. Psychiatr Clin North Am 1998;21:443-63.

56. Howard LM, Oram S, Galley H, et al. Domestic violence and perinatal mental disorders: a systematic review and meta-analysis. PLoS Med 2013;10:e1001452.

57. Ludermir AB, Lewis G, Valongueiro SA, et al. Violence against women by their intimate partner during pregnancy and postnatal depression: a prospective cohort study. Lancet 2010;376:903-10.

58. Halim N, Beard J, Mesic A, et al. Intimate partner violence during pregnancy and perinatal mental disorders in low and lower middle income countries: A systematic review of literature, 1990-2017. Clin Psychol Rev 2017.

59. Boden JM, Fergusson DM. Alcohol and depression. Addiction 2011:106:906-14.

60. Machisa MT, Christofides N, Jewkes R. Mental ill health in structural pathways to women's experiences of intimate partner violence. PLOS One 2017; 12:0175240.

61. Foran HM, O'Leary KD. Alcohol and intimate partner violence: a meta-analytic review. Clin Psychol Rev 2008;28:1222-34.

62. Leonard KE, Eiden RD. Marital and family processes in the context of alcohol use and alcohol disorders. Annu Rev Clin Psychol 2007;3:285-310.

63. Leadley K, Clark CL, Caetano R. Couples' drinking patterns, intimate partner violence, and alcohol-related partnership problems. J Subst Abuse 2000;11:253-63.

64. Barbosa C, Taylor B, Godfrey C, et al. Modelling lifetime QALYs and health care costs from different drinking patterns over time: a Markov model. Int J Methods Psychiatr Res 2010;19:n/a-109.

65. Room R, Babor T, Rehm J. Alcohol and public health. The Lancet 2005;365:519-30.

66. Campbell JC. Health consequences of intimate partner violence. Lancet 2002;359:1331-6.

67. Johnson MP. Patriarchal terrorism and common couple violence: two forms of violence against women. J Marriage Fam 1995;57:283-94.

68. Nyamayemombe C, Mishra V, Rusakaniko S, et al. The association between violence against women and hiv: evidence from a national population-based survey in Zimbabwe. Calverton, Maryland: ICF Macro, 2010

69. Cohen S, Kamarck T, Mermelstein R. A global measure of perceived stress. J Health Soc Behav 1983;24:385-96. 